\title{
Avaliação da qualidade e do entendimento das Gestantes e Puérperas na Atenção Primária no Pré-natal
}

Evaluation of the quality and understanding of pregnant women and mothers in primary care in prenatal care

Evaluación de la calidad y comprensión de las gestantes y madres en atención primaria en atención prenatal

Recebido: 26/01/2021 | Revisado: 01/02/2021 | Aceito: 05/02/2021 | Publicado: 10/02/2021

Dayane Lima Caldeira da Silva

ORCID: https://orcid.org/0000-0001-8895-5183 Instituição Brasileira de Medicina e Reabilitação, Brasil E-mail:daylimacs@gmail.com

Gabriel Conde y Martin Cebriano

ORCID: https://orcid.org/0000-0002-0125-7874 Instituição Brasileira de Medicina e Reabilitação, Brasil E-mail:cebrianogabriel@gmail.com

Helga Rocha Pitta Portella Figueiredo

ORCID: https://orcid.org/0000-0003-2899-1988 Instituição Brasileira de Medicina e Reabilitação, Brasil E-mail: helgapitta@gmail.com

Isabelle Cristiny Gregorio Amorim Passos

ORCID: https://orcid.org/0000-0002-4360-2197 Instituição Brasileira de Medicina e Reabilitação, Brasil E-mail: belle_jpa@hotmail.com

Karen Lorrana Reis Barbosa

ORCID: https://orcid.org/0000-0003-2546-6299 Instituição Brasileira de Medicina e Reabilitação, Brasil E-mail:karenlorranar@gmail.com

Paloma Pereira de Andrade

ORCID: https://orcid.org/0000-0002-6034-6244 Instituição Brasileira de Medicina e Reabilitação, Brasil E-mail:paloma.crvg115@gmail.com

Thiago Nascimento Sousa Santos

ORCID: https://orcid.org/0000-0002-7887-5021 Instituição Brasileira de Medicina e Reabilitação, Brasil E-mail:thiagonasciment@icloud.com

Victória Emília Leitão Santiago

ORCID: https://orcid.org/0000-0002-1973-1060 Instituição Brasileira de Medicina e Reabilitação, Brasil E-mail:santiago.viccc@gmail.com

\begin{abstract}
Resumo
O objetivo visa apresentar a importância de um pré-natal de qualidade, de como esta gestante recebe as informações e se recebe, e sobre o impacto das informações passadas durante esse período. Trata-se de um estudo de revisão integrativa, de natureza qualitativa, que proporciona de uma forma ampla o conhecimento e a incorporação da aplicabilidade de artigos anteriores com assuntos que apresentam hipóteses idênticas ou relacionadas ao tema. Para levantamento dos dados, foram utilizadas as palavras-chave e alguns critérios de inclusão, como: ter coleta de dados que apresentem a visão da gestante durante o ciclo gravídico puerperal e estudo que tenha sido realizado em âmbito nacional. E como resultado selecionamos para o estudo 14 artigos relacionados ao tema e posteriormente classificados em duas categorias: percepção da gestante e/ou puérperas e, qualidade da assistência. Mesmo com alguns pontos positivos, concluímos que, os estudos apresentados destacam que ainda há necessidade de melhorias na estratégia, promoção e educação em saúde no atendimento básico ao pré-natal para que tenhamos efetividade e qualidade adequada em todo o Brasil. Sendo assim, é indubitável que ainda exista um déficit em relação à qualidade da assistência na Atenção Primária à Saúde (APS) à gestante e puérpera. Por esse motivo, sugerimos utilizar a tecnologia atual a nosso favor para incentivo e também, a realização de mais estudos e revisões com estes cenários. Palavras-chave: Gestantes; Puerpério; Educação em saúde; Cuidado pré-natal.
\end{abstract}




\begin{abstract}
The objective is to present the importance of quality prenatal care, how this pregnant woman receives information and is received, and about the impact of information passed during this period. It is an integrative review study, of a qualitative nature, which broadly provides the knowledge and incorporation of the applicability of previous articles with subjects that present identical or related hypotheses. For data collection, keywords and some inclusion criteria were used, such as: having data collection that present the pregnant woman's vision during the puerperal pregnancy cycle and a study that has been carried out at the national level. As a result, we selected 14 articles related to the topic for the study and later classified into two categories: perception of pregnant women and / or puerperal women and quality of care. Even with some positive points, we conclude that the studies presented highlight that there is still a need for improvements in the strategy, promotion and health education in basic prenatal care so that we have effectiveness and adequate quality throughout Brazil. Therefore, there is no doubt that there is still a deficit in relation to the quality of care in Primary Health Care (PHC) for pregnant women and women who have recently given birth. For this reason, we suggest using current technology in our favor for incentive and also, conducting further studies and reviews with these scenarios.
\end{abstract}

Keywords: Pregnant women; Puerperium; Health education; Prenatal care.

\title{
Resumen
}

El objetivo es presentar la importancia de la atención prenatal de calidad, cómo esta gestante recibe información y es recibida, y sobre el impacto de la información transmitida durante este período. Se trata de un estudio de revisión integradora, de carácter cualitativo, que proporciona de manera amplia el conocimiento e incorporación de la aplicabilidad de artículos anteriores con temas que presentan hipótesis idénticas o afines. Para la recolección de datos se utilizaron palabras clave y algunos criterios de inclusión, tales como: tener recolección de datos que presenten la visión de la gestante durante el ciclo del embarazo puerperal y un estudio que se ha realizado a nivel nacional. Como resultado, se seleccionaron 14 artículos relacionados con el tema para el estudio y luego se clasificaron en dos categorías: percepción de la gestante y / o puerperal y calidad de la atención. Incluso con algunos puntos positivos, concluimos que los estudios presentados resaltan que aún existe la necesidad de mejoras en la estrategia, promoción y educación en salud en la atención prenatal básica para que tengamos efectividad y calidad adecuada en todo Brasil. Por tanto, no cabe duda de que aún existe un déficit en relación a la calidad de la atención en Atención Primaria de Salud (APS) para las embarazadas y las que han dado a luz recientemente. Por este motivo, sugerimos utilizar la tecnología actual a nuestro favor como incentivo y también, realizar más estudios y revisiones con estos escenarios.

Palabras clave: Mujeres embarazadas; Puerperio; Educación para la salud; Cuidado prenatal.

\section{Introdução}

\section{1 Pré-natal}

O pré-natal é o acompanhamento feito pela mulher em seu período gravídico-puerperal, em que se é realizado exame físico e complementar a fïm de monitorar a saúde materno fetal. A gestação é um acontecimento que muda a estrutura da mulher fisiologicamente e afeta seu desenvolvimento psico-físico-social. Nas consultas subsequentes ao descobrimento da gravidez, iremos abordar com essas mulheres, fatores importantes que vão acontecer e impactar na vida delas, criando sempre um vínculo com a paciente e seu acompanhante, abordando sobre como essa mulher vai ter o apoio necessário tanto psicológico quanto social, no seu período gestacional e no puerpério. É de extrema importância estar atento e se mostrar ouvinte, para tirar dúvidas e preservar esse momento tão especial, certificando que os recursos físicos, materiais, humanos e técnicos sejam prestados durante o período pré-natal.

\subsection{Atuação do enfermeiro}

A Unidade Básica de Saúde (UBS) é o principal meio de acolher essa mulher no Sistema Único de Saúde (SUS). É neste meio, que vamos aproveitar para colocar em prática as estratégias de promoção em saúde para a gestante e seu bebê garantindo a integralidade do cuidado, como prevê nas diretrizes do SUS.

Os Enfermeiros mediante as suas atividades exercem um papel importante junto às gestantes para que a adesão ao Prénatal seja contínua (Rio de Janeiro, 2012, p. 70). 
A base legal que fundamenta a atuação do profissional enfermeiro está respaldada pela Lei do Exercício Profissional de Enfermagem $n^{\circ}$ 7.498/86, Resolução do Conselho Federal de Enfermagem (Cofen) n $n^{\circ} 195 / 1997, n^{\circ} 223 / 1999, n^{\circ}$ 271/2002 e n⿳2 272/2002, Pareceres Cofen no 040/95 e n⿳ 15/97, e Portaria n $1459 / 2011$ que institui a Rede Cegonha.

Assim, cabe ao enfermeiro da atenção primária em saúde acompanhar o pré-natal de baixo risco e o puerpério, conforme garantido pela Lei do exercício Profissional, regulamentada pelo Decreto nº 94.406/87. O enfermeiro da atenção primária em saúde, além da competência para atuar na atenção à saúde da mulher, cabe-lhe promover ações educativas que busquem atender a mulher na sua perspectiva de gênero, na análise de seu perfil epidemiológico e no planejamento de ações de saúde, que tenham como objetivo promover a melhoria das condições de vida, a igualdade e os direitos de cidadania da mulher, sua família e comunidade (Rio de Janeiro, 2017, p. 100).

Nas primeiras consultas do pré-natal (primeiro trimestre) o profissional de saúde (enfermeiros e médicos), deverá realizar a anamnese abordando aspectos epidemiológicos, exame físico completo, preparação e cuidados com a mama, hemograma, testes rápidos de triagem para Sífilis e/ou VDRL, HIV, toxoplasmose IgG e IgM, sorologia para Hepatite B, urocultura+sumário de urina e Ultrassonografia. Se houver alguma indicação clínica, realizar exame de cultura colpocitológica, de secreção vaginal e parasitológico de fezes. Esses exames iniciais, tem como objetivo identificar possíveis infecções e prevenir a gestante e o feto de prováveis doenças ou complicações que possa acarretar uma gravidez de alto risco, sofrimento fetal e até mesmo o aborto. De 0 a 28 semanas de gestação, é realizada uma consulta por mês. No segundo trimestre de gestação, o exame físico e a anamnese também deverão ser realizados e caso haja suspeita ou risco para Diabetes gestacional, realizar o teste de intolerância para glicose. Da $28^{\mathrm{a}}$ até a $36^{\mathrm{a}}$ semana, as consultas deverão ser realizadas quinzenalmente. No terceiro e último trimestre, os exames da primeira consulta serão repetidos. Da $36^{\mathrm{a}}$ à $41^{\mathrm{a}}$ semana, as consultas são realizadas semanalmente.

É importante ressaltar que em todas as consultas o exame físico é também composto pela medição da altura uterina, idade gestacional, peso da gestante, verificação da pressão arterial, avaliação dos batimentos cardiofetais (pinard e/ou sonar) e manobra de Leopold (para observação da posição fetal). Além de todas as informações passadas em todas as consultas pré-natais, a caderneta da gestante que é disponibilizada na primeira consulta contém todas as informações necessárias que a mulher precisa saber nesse período, como: direitos garantidos por lei, inclusive sobre entrega para adoção, como o bebê se forma, cuidados importantes nutricionais, sexo, sintomas, entre outros. Essa caderneta também possui o gráfico de acompanhamento nutricional, curva de altura uterina e idade gestacional, esquemas de exames e vacinas, onde são preenchidos todos os dados coletados.

O plano de parto também é aplicado no pré-natal, tem como objetivo preparar a gestante para os próximos passos, passando todo conhecimento necessário para a gestante se sentir segura e confiante. Abordando sobre seus desejos, métodos não farmacológicos, visitas ao local do parto, exercícios durante o trabalho de parto, posições de livre escolha e preparação da bolsa do bebê. A amamentação, é um passo fundamental do plano de parto, ensinar a mulher o posicionamento correto do bebê, a pega certa e a troca dos seios entre as mamadas alternadas. É essencial, explicar para a mulher como funciona o trabalho de parto, o início das contrações e a evolução delas. Saber contar o tempo de contração, para ir ao hospital no momento certo, sendo importante para evitar iatrogenias e deslocamentos desnecessários.

\subsection{Puerpério}

O puerpério é o período do pós parto, que dura em torno de 45 a 60 dias, popularmente chamado de resguardo. Nesse tempo, é preciso acompanhar esta mulher para avaliar sua involução uterina, como esta quantidade dos Lóquios, suas eliminações fecais e alimentação, se a amamentação está sendo feita corretamente e observar qualquer sinal de inflamação, tanto mamária quanto vaginal. Esclarecer sobre a pausa da vida sexual entre 30 dias ou até a cicatrização durante esse momento, é essencial para uma boa recuperação. Na avaliação é importante considerar o estado psico-emocional-social desta mulher, como o acompanhante está se envolvendo e participando deste período, se possui apoio social familiar e sobre sua relação com o recémnascido. Esses fatores, possuem bastante impacto sendo uma fase complexa, podendo causar depressão pós parto. 
Durante a graduação passamos pela matéria de saúde da mulher e nos interessamos com o assunto abordado sobre educação em saúde aplicado no pré-natal. Logo, identificamos que amigos e parentes próximos passaram pelo período gravídico puerperal e não tinham tais conhecimentos básicos. Com isso, surgiu o entusiasmo de realizar uma pesquisa que mostrasse o real déficit dessa qualidade em educação em saúde na atenção primária voltada ao pré-natal.

Este trabalho visa apresentar a importância de um pré-natal de qualidade, de como está gestante recebe as informações e se recebe, e sobre o impacto das informações passadas durante esse período. Ponderar o serviço prestado pode-se aperfeiçoar e ajustar o que de fato está sendo feito no acompanhamento pré-natal. A educação em saúde é um ponto chave que possui grande impacto e relevância para a melhora desse cuidado com a sociedade.

Este trabalho tem como objetivo, realizar busca pela produção científica sobre a qualidade da assistência e a percepção da gestante no pré-natal na APS brasileira.

\section{Metodologia}

Trata-se de um estudo de revisão integrativa, de natureza qualitativa, segundo Pereira A.S. et al. (2018) que proporciona de uma forma ampla o conhecimento e a incorporação da aplicabilidade de artigos anteriores com assuntos que apresentam hipóteses idênticas ou relacionadas ao tema.

Para o levantamento dos artigos na literatura, realizou-se uma busca na base de dados Scielo, durante o período de setembro e Outubro de 2020.

Os critérios de inclusão definidos para a seleção dos artigos foram: artigos publicados em português, inglês e espanhol; artigos na íntegra que retratam a temática referente à revisão integrativa e artigos publicados e indexados nos referidos bancos de dados nos últimos dez anos. Utilizamos como critério de exclusão, artigos produzidos fora do território brasileiro.

Foram utilizados, para busca dos artigos, os seguintes descritores e suas combinações nas línguas portuguesa e inglesa: "Gestantes", "Puerpério", "Educação em saúde” e "Cuidado pré-natal”.

Figura 1: Fluxograma de busca de artigos.

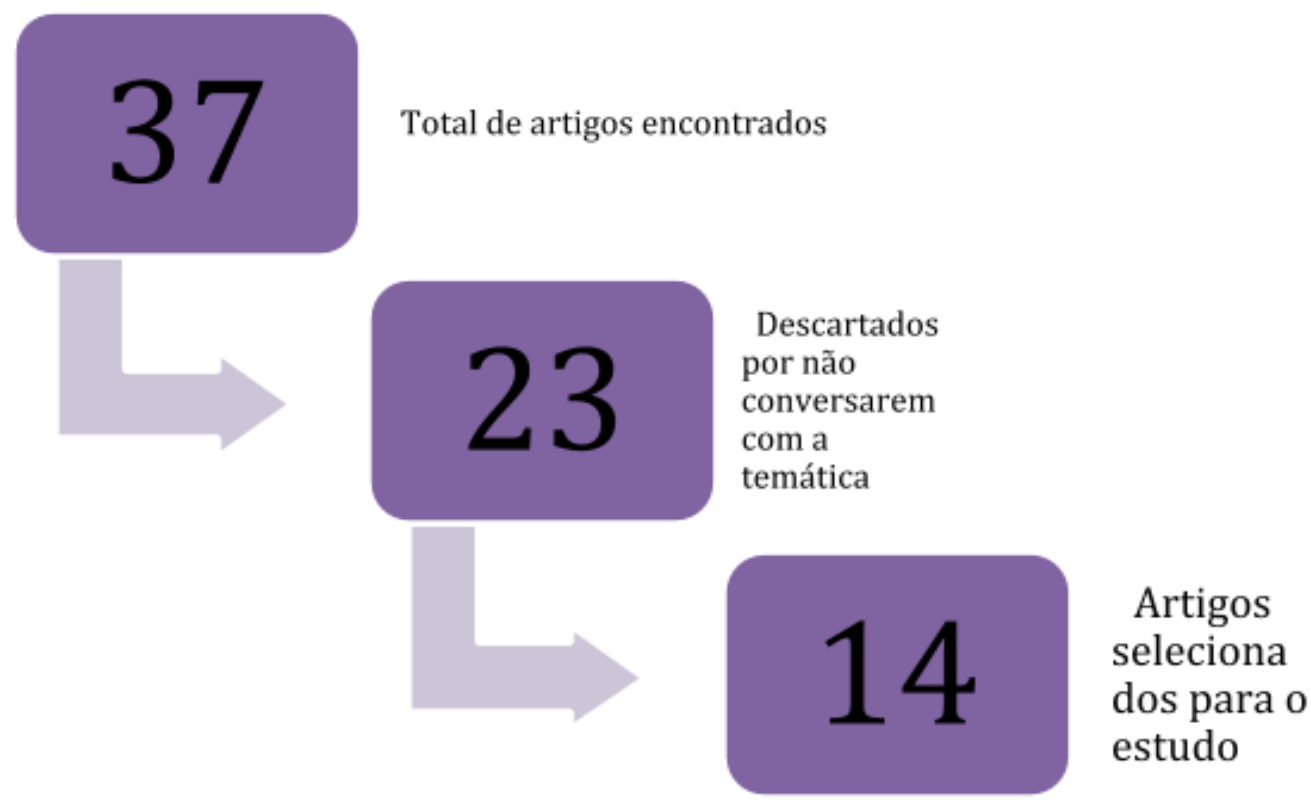

Fonte: Autores.

Avaliando o fluxograma, obtivemos um total de 37 artigos encontrados. Dentre eles descartamos 23 por não coincidirem com o tema abordado e por fim, foram selecionados 14 artigos para o estudo (Figura 1). 


\section{Resultados}

Os critérios utilizados para inclusão dos artigos na revisão foram: ter coleta de dados que apresentem a visão da gestante durante o ciclo gravídico puerperal; Se o pré-natal foi realizado com todas as etapas que o qualifiquem sendo de qualidade ou não; Se a educação em saúde foi proporcionada durante o estudo de coleta de dados ou apresentada de forma secundária em uma revisão e que tenha sido realizado em âmbito nacional.

Quadro 1: Artigos selecionados para o estudo.

\begin{tabular}{|c|c|c|c|c|c|}
\hline № & Título & Autores & Ano & $\begin{array}{l}\text { Categoria do assunto } \\
\text { principal }\end{array}$ & $\begin{array}{l}\text { Tipo de } \\
\text { estudo }\end{array}$ \\
\hline 1 & $\begin{array}{l}\text { Percepções de gestantes acerca do } \\
\text { cuidado pré-natal na atenção primária à } \\
\text { saúde. }\end{array}$ & $\begin{array}{c}\text { Livramento, D.V.P et } \\
\text { al. }\end{array}$ & 2019 & Saúde da mulher & Qualitativo \\
\hline 2 & $\begin{array}{c}\text { Avaliação do processo na assistência } \\
\text { pré-natal de gestantes com risco } \\
\text { habitual }\end{array}$ & Balsells, M.M.D et al. & 2018 & Saúde da mulher & Qualitativo \\
\hline 3 & $\begin{array}{c}\text { Avaliação do cuidado pré-natal na } \\
\text { atenção primária a saúde na percepção } \\
\text { da gestante }\end{array}$ & $\begin{array}{l}\text { Prudêncio, P.S; } \\
\text { Mamede, F.V. }\end{array}$ & 2018 & Saúde da mulher & Transversal \\
\hline 4 & $\begin{array}{c}\text { Qualidade da atenção pré-natal na rede } \\
\text { básica de saúde do Brasil: indicadores } \\
\text { e desigualdades sociais }\end{array}$ & Tomasi, E et al. & 2016 & Educação em saúde & Transversal \\
\hline 5 & $\begin{array}{l}\text { Orientações às gestantes no pré-natal: } \\
\text { a importância do cuidado compartilhado } \\
\text { na atenção primária em saúde }\end{array}$ & Marques, B.L et al. & 2020 & Educação em saúde & $\begin{array}{l}\text { Quantitativo- } \\
\text { transversal }\end{array}$ \\
\hline 6 & $\begin{array}{l}\text { Assistência pré-natal na percepção de } \\
\text { puérperas provenientes de diferentes } \\
\text { serviços de saúde }\end{array}$ & $\begin{array}{l}\text { Ceron, M.I; Barbieri, A } \\
\text {; Fonseca, L.M; } \\
\text { Fedosse, E. }\end{array}$ & 2012 & Educação em saúde & Transversal \\
\hline 7 & $\begin{array}{l}\text { Adequabilidade da assistência pré-natal } \\
\text { em uma estratégia de saúde da família } \\
\text { de porto alegre-rs }\end{array}$ & $\begin{array}{l}\text { HASS, C.N; } \\
\text { TEIXEIRA, L.B; } \\
\text { BEGHETTO, M.G. }\end{array}$ & 2013 & Educação em saúde & Quantitativo \\
\hline 8 & $\begin{array}{l}\text { Processo e resultado do cuidado pré- } \\
\text { natal segundo os modelos de atenção } \\
\text { primária: estudo de coorte }\end{array}$ & $\begin{array}{l}\text { Oliveira, R.L.A; } \\
\text { Ferrari, A.P; Parada, } \\
\text { C.M.G.L. }\end{array}$ & 2019 & Saúde da mulher & Quantitativo \\
\hline 9 & $\begin{array}{c}\text { Educação em saúde no ciclo gravídico- } \\
\text { puerperal: sentidos atribuídos por } \\
\text { puérperas }\end{array}$ & Guerreiro,E.M et al. & 2014 & Educação em saúde & $\begin{array}{l}\text { Qualitativo e } \\
\text { descritivo }\end{array}$ \\
\hline 10 & $\begin{array}{c}\text { Opinião das gestantes sobre } \\
\text { acompanhamento da enfermeira } \\
\text { obstetra no pré-natal de um hospital } \\
\text { universitário }\end{array}$ & $\begin{array}{l}\text { Spindola, T; Progianti, } \\
\text { J.M; Penna, L.H.G. }\end{array}$ & 2012 & Saúde da mulher & $\begin{array}{l}\text { Exploratório } \\
\text { descritivo }\end{array}$ \\
\hline 11 & $\begin{array}{c}\text { Percepção das puérperas sobre a } \\
\text { assistência prestada pela equipe de } \\
\text { saúde no pré-natal }\end{array}$ & Vieira, S.M et al. & 2011 & Saúde da mulher & $\begin{array}{l}\text { Qualitativo } \\
\text { descritivo }\end{array}$ \\
\hline 12 & Assistência pré-natal no Brasil & Viellas, E.F et al. & 2014 & Educação em saúde & $\begin{array}{l}\text { Qualitativo } \\
\text { descritivo }\end{array}$ \\
\hline
\end{tabular}




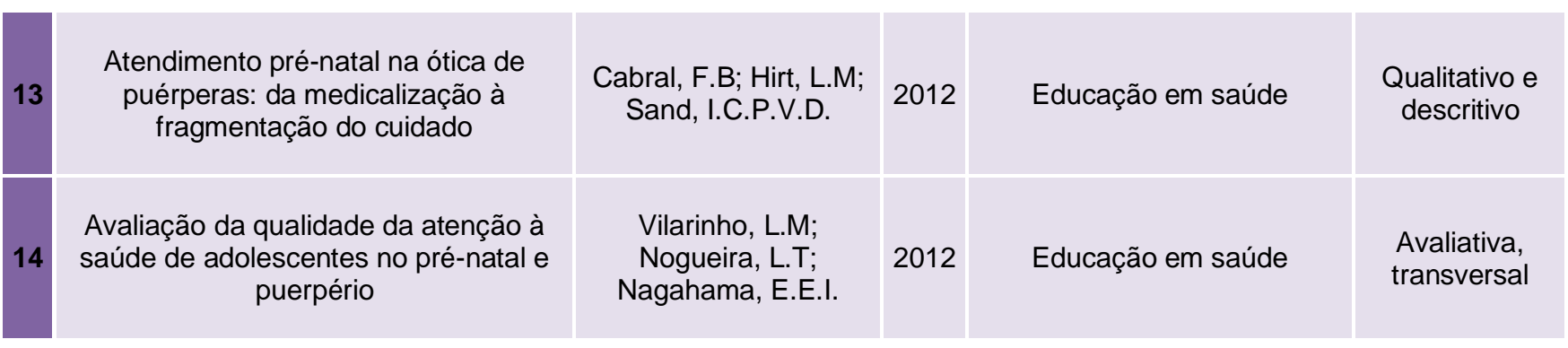

Fonte: Autores.

Podemos observar no Quadro 1, os artigos selecionados. Separamos e numeramos cada um deles para podermos segmentá-los e discuti-los em tópicos separados, tendo uma melhor visibilidade.

\section{Discussão}

Foram avaliados um a um, os artigos e posteriormente optamos em classificar em duas categorias, para melhor interpretação. Sendo elas: percepção da gestante e/ou puérperas e, qualidade da assistência.

\subsection{Percepção da gestante e/ou puérpera}

Cinco dos artigos selecionados relataram a visão da gestante/puérpera no acolhimento pré-natal, com utilização de entrevistas diretas com as mulheres entre 16-46 anos através de formulários previamente estruturados. Com isso, foram avaliados o real conhecimento e informações passadas durante a assistência no pré-natal. Alguns temas estão visivelmente destacados por falta de promoção e educação em saúde, acolhimento, comunicação e cuidado humanizado 1, 6, 10,11 e 13.

Na promoção e educação à saúde foi percebido pelas mulheres a falta de materiais, atividades em grupo, quantidade e qualidade insuficiente de consultas, sendo esses métodos poderosos para reduzir agravos na gestação, anular dúvidas referentes aos períodos de amamentação, parto, pós-parto e até mesmo inseguranças do dia a dia.

Foram observados questões mais levantadas e abordadas pelos profissionais, sendo eles assuntos relacionados a alimentação, uso de substâncias tóxicas e álcool. Mesmo com tais informações passadas, identifica-se que as mulheres entrevistadas não se sentem seguras durante a gestação, o que ocasiona até a busca de informações por meios tecnológicos e/ou experiência vividas por outras mães. Com isso, podemos identificar notoriamente como a falta de informação gera uma cadeia de incertezas, medos e até possíveis iatrogenias. A dificuldade na comunicação das pacientes com os profissionais médicos, também foi relevante e explícito. Vemos como é importante criar um ambiente agradável e acolhedor nas consultas para que as mulheres vivenciem um pré-natal adequado e seguro.

Com relação a exames solicitados, notou-se um desconforto no geral com o excesso de exames de imagens sem necessidade.

Comumente, a preocupação dos profissionais deu mais enfoque para o bebê do que com a mãe em si, o que afeta emocionalmente e psicologicamente as mulheres durante todo o ciclo gravídico-puerperal.

Como ponto positivo, algumas dessas mulheres se sentiram satisfeitas, confiante e à vontade quanto ao atendimento contínuo realizados pelos mesmos profissionais, na maioria, sendo esses atendimentos feitos por enfermeiros.

\subsection{Qualidade da assistência}

Os nove artigos que descrevem sobre a qualidade da assistência, usam formulários precedentemente estruturados para investigar se há qualidade na assistência ou não. Em cada um dos estudos é perceptível a porcentagem negativa da avaliação do cuidado. A baixa aquisição do pré-natal e consultas puerperais é correlacionado ao não entendimento da importância do mesmo, 
tendo em vista que, as unidades não realizam a promoção do cuidado para sanar essa insuficiência de atendimento. Sem pormenorizar os artigos, identificamos que são realizadas apenas o mínimo de consultas pré-natais, e que apenas exames laboratoriais simples, vacinas e orientações sobre riscos, apresentaram-se de forma abundante. Complementou-se também, que informações passadas às mulheres sobre parto e amamentação foram transcorridas inadequadamente, entretanto com baixo percentual de acontecimentos. Integralmente os artigos declaram que a maior proporção de avaliação da assistência, indicou que não houve qualidade na assistência do pré-natal $2,3,4,5,7,8,9,12 \mathrm{e} 14$.

A baixa adequação ao pré-natal foi um fator relevante entre os artigos, tendo como fatores gerais o baixo índice de consultas precoces, consultas puerperais, exames laboratoriais incompletos e desinformação.

Exame físico completo ocorreu também em baixo índice, sendo realizado somente a aferição de pressão arterial e altura uterina. Observou-se ainda, que não houve diferença de atendimento entre as UBS (Unidades Básicas de Saúde) e USF (Unidades de Saúde da Família).

Os atendimentos feitos por apenas um tipo de profissional foram os que menos tiveram orientações, como por exemplo sobre visita a maternidades, parto e amamentação, entretanto foram mais alertados aos sinais de riscos. As consultas que foram intercaladas entre profissionais médicos e profissionais enfermeiros, tanto no pré-natal quanto no puerpério, obteve maior êxito na assistência e no vínculo de orientações passadas, comparadas aquelas que se deu por apenas um destes profissionais.

No puerpério, obteve-se maior número de não adequação às consultas, ou seja, não houve retorno até o $42^{\circ}$ dia de pósparto e também não teve visita domiciliar.

Mulheres acompanhadas de familiares possuem mais adesão e participação no pré natal, diferente de mulheres que não possuem parceiros ou apoio familiar, nesses casos de desacompanhamento a maioria dá início ao atendimento tardiamente.

\section{Conclusão}

Os resultados dos estudos apresentados, apontam a necessidade de melhorias na educação e promoção em saúde, assim como a estratégia de estimular a mulher a procurar serviços de apoio e fazer com que a participação seja mais ativa. Faz-se necessário também, impulsionar os profissionais para que sejam mais persistentes na dedicação de atividades e estratégias. Foi possível perceber a compreensão das gestantes em relação ao cuidado recebido e é notável como a qualidade da assistência é debilitada, podendo-se converter de forma positiva para se aperfeiçoar. Devemos nos atentar também, ao fato de passar informações adequadas e atualizadas, para que não haja uma cadeia de má informação entre mulheres. É inquestionável, que ainda existem carências em relação à qualidade da assistência na APS, à gestante e à puérpera.

Neste contexto, sugerimos trabalhar a interação profissional x paciente, aproveitando a tecnologia atual como forma de incentivo para a mulher gestante tirar suas dúvidas e receber informações de educação em saúde voltadas à temática do pré-natal e puerpério. Apontamos também, a importância de realizar mais estudos e revisões com estes cenários, para que possamos entender tais necessidades e trabalhar em cima delas, mantendo as informações sempre atualizadas.

\section{Referência}

Balsells, M. M. D., Oliveira, T. M. F., Bernardo, E. B. R., Aquino, P. S., Damasceno, A. K. C., Castro, R. C. M. B., \& Pinheiro, A. K. B. (2018). Avaliação do processo na assistência pré-natal de gestantes com risco habitual. Acta Paul Enferm, 31(3), 247-54. https://www.scielo.br/pdf/ape/v31n3/1982-0194-ape-31-030247.pdf. 10.1590/1982- 0194201800036

Cabral, F. B., Hirt, L. M., \& Sand, I. C. P. V. D. (2012). Atendimento pré-natal na ótica de puérperas: da medicalização à fragmentação do cuidado. Rev Esc Enferm, 47(2), 281-7. https://www.scielo.br/pdf/reeusp/v47n2/02.pdf

Ceron, M. I., Barbieri, A., Fonseca, L. M., \& Fedosse, E. (2012). Assistência pré-natal na percepção de puérperas provenientes de diferentes serviços de saúde. Revista CEFAC, 15(3), 653-662. https://www.scielo.br/pdf/rcefac/v15n3/184-11.pdf 
Research, Society and Development, v. 10, n. 2, e20810212385, 2021

(CC BY 4.0) | ISSN 2525-3409 | DOI: http://dx.doi.org/10.33448/rsd-v10i2.12385

Guerreiro, E. M., Rodrigues, D. P., Queiroz, A. B. A., \& Ferreira, M. A. (2014). Educação em saúde no ciclo gravídico-puerperal: sentidos atribuídos por puérperas. Revista Brasileira de Enfermagem, 67(1), 13-21. https://www.scielo.br/pdf/reben/v67n1/0034-7167-reben-67-01-0013.pdf. 10.5935/00347167.20140001

Hass, C. N., Teixeira, L. B., \& Beghetto, M. G. (2013). Adequabilidade da assistência pré-natal em uma estratégia de saúde da família de porto alegre-rs. Revista Gaúcha de Enfermagem, 34(3), 22-30. https://www.scielo.br/pdf/rgenf/v34n3/a03v34n3.pdf

Livramento, D. V. P., Backes, M. T. S., Damiani, P. R., Castillo, L. D. R., Backes, D. S., \& Simão, A. M. S. (2019). Percepções de gestantes acerca do cuidado pré-natal na atenção primária à saúde. Rev Gaúcha Enferm, 40, e20180211. https://www.scielo.br/pdf/rgenf/v40/1983-1447-rgenf-40-e20180211.pdf. $10.1590 / 1983-1447.2019 .2018021$

Marques, B. L., Tomasi, Y. T., Saraiva, S. S., Boing, A. F., \& Geremia, D. S. (2020). Orientações às gestantes no pré-natal: a importância do cuidado compartilhado na atenção primária em saúde. Escola Anna Nery Revista de Enfermagem, 25(1), 2020-0098. https://www.scielo.br/pdf/ean/v25n1/1414-8145ean-25-1-e20200098.pdf. 10.1590/2177-9465-ean-2020-0098

Pereira, A. S., Shitsuka, D. M., Pereira, F. J., \& Shitsuka, R. (2018). Metodologia da pesquisa científica. Ed. UAB/NTE/UFSM. https://repositorio.ufsm.br/bitstream/handle/1/15824/Lic_Computacao_Metodologia-Pesquisa-Cientifica.pdf?sequence=1.

Oliveira, R. L. A., Ferrari, A. P., \& Parada, C. M. G. L. (2019). Processo e resultado do cuidado pré-natal segundo os modelos de atenção primária: estudo de coorte. Revista Latino-Americana de enfermagem, 27, e3058. https://www.scielo.br/pdf/rlae/v27/1518-8345-rlae-27-e3058.pdf. 10.1590/1518-8345.2806.3058

Protocolo de Enfermagem na Atenção Primária (Ed.) (2017) https://issuu.com/asasrio/docs/protocoloenfermagem_saudemental_ord

Protocolos de Enfermagem na Atenção Primária à Saúde (Ed 2a) (2012) https://www.rio.rj.gov.br/dlstatic/10112/4446958/4111921/enfermagem.pdf

Prudêncio, P. S., \& Mamede, F. V. (2018). Avaliação do cuidado pré-natal na atenção primária a saúde na percepção da gestante. Rev Gaúcha Enferm, 39, e20180077. https://www.scielo.br/pdf/rgenf/v39/1983-1447-rgenf-39-04-e20180077.pdf. 10.1590/1983- 1447.2018.20180077

Spindola, T., Progianti, J. M., \& Penna, L. H. G. (2012). Opinião das gestantes sobre acompanhamento da enfermeira obstetra no pré-natal de um hospital universitário. Ciencia y Enfermeria XVIII, (2), 65-73. https://scielo.conicyt.cl/pdf/cienf/v18n2/art_07.pdf

Tomasi, E., Fernandes, P. A. A., Fischer, T., Siqueira, F. C. V., Silveira, D. S., Thumé, E., \& Facchini, L. A. (2016). Qualidade da atenção pré-natal na rede básica de saúde do Brasil: indicadores e desigualdades sociais. Cad Saúde Pública, 33(3), e00195815. https://www.scielo.br/pdf/csp/v33n3/1678-4464-csp-3303-e00195815.pdf. 10.1590/0102-311X00195815

Vieira, S. M., Bock, L. F., Zocche, D. A., \& Pessota, C. U. (2011). Percepção das puérperas sobre a assistência prestada pela equipe de saúde no pré-natal. Texto Contexto Enferm, 20, 255-62. https://www.scielo.br/pdf/tce/v20nspe/v20nspea32.pdf

Viellas, E. F., Domingues, R. M. S. M., Dias, M. A. B., Gama, S. G. N., Filha, M. M. T., Costa, J. V., \& Leal, M. C. (2014). Assistência pré-natal no Brasil. Cad Saúde Pública, 30, S85-S100. https://www.scielo.br/pdf/csp/v30s1/0102-311X-csp-30-s1-0085.pdf. 10.1590/0102-311X00126013

Vilarinho, L. M., Nogueira, L. T., \& Nagahama, E. E. I. (2012). Avaliação da qualidade da atenção à saúde de adolescentes no pré-natal e puerpério. Esc Anna Nery, 16(2), 312-319. https://www.scielo.br/pdf/ean/v16n2/15.pdf 